\title{
NORMABILITY OF CERTAIN TOPOLOGICAL RINGS
}

\author{
SETH WARNER ${ }^{1}$
}

\begin{abstract}
Criteria are given for the normability of certain topological rings. These criteria yield yeneralizations of known theorems on the normability of compact integral domains and topological fields.
\end{abstract}

In [6, Theorem 7], Z. S. Lipkina proved that the topology of a metrizable, compact ring $A$ with identity and without zero-divisors is given by a norm, i.e., a function \|\| from $A$ into the nonnegative real numbers satisfying $\|x\|=0$ if and only if $x=0,\|-x\|=\|x\|,\|x+y\| \leqq\|x\|+\|y\|$, and $\|x y\| \leqq\|x\|$ i|y $y$ for all $x, y \in A$. Her proof depended upon exhibiting $A$ as a quotient ring of a certain power series ring in countably many noncommuting variables over a coefficient ring whose elements did not, however, commute with the variables. Here we shall give an elementary proof of a generalization of her result concerning normability.

A function $v$ defined on a ring $A$ is a natural pseudovaluation if the range of $v$ is contained in the natural numbers together with $+\infty$ and if for all $x, y \in A, v(x)=+\infty$ if and only if $x=0, v(-x)=v(x), v(x+y) \geqq$ $\min \{v(x), v(y)\}$, and $v(x y) \geqq v(x)+v(y)$. Clearly the exponential of a natural pseudovaluation to a base $<1$ is a nonarchimedean norm satisfying $\|x\| \leqq 1$ for all $x \in A$. An ideal $a$ in a topological ring is topologically nilpotent if the filter base formed by its powers converges to zero.

THEOREM 1. The topology of a topological ring $A$ is defined by a natural pseudovaluation if and only if the following three conditions hold:

$1^{\circ} A$ is metrizable.

$2^{\circ}$ The open ideals of $A$ form a fundamental system of neighbornoods of zero.

$3^{\circ} A$ contains an open, topologically nilpotent ideal.

Proof. Necessity. Let $a_{n}=\{x \in A: v(x) \geqq n\}$. Then $\left\{a_{n}\right\}_{n \geqq 1}$ is a fundamental system of neighborhoods of zero, each $a_{n}$ is an ideal, and $\mathfrak{a}_{1}^{n} \subseteq \mathfrak{a}_{n}$ for all $n \geqq 1$.

Received by the editors June 16, 1971.

AMS 1970 subject classifications. Primary 46H05, 16 A80.

Key words and phrases. Norm, pseudovaluation, topological ring, compact ring.

1 Written while the author was in residence at Reed College. 
Sufficiency. Let $r$ be an open, topologically nilpotent ideal; by $1^{\circ}$ and $2^{\circ}$ there is a fundamental decreasing sequence $\left\{a_{k}\right\}_{k \geqq 1}$ of ideal neighborhoods of zero each contained in $\mathfrak{r}$. For each $n \geqq 1$ let $\mathfrak{b}_{n}=\sum \mathfrak{a}_{k_{1}} \mathfrak{a}_{k_{2}} \cdots \mathfrak{a}_{k_{r}}$, the sum extending over all $r$-tuples $\left(k_{1}, k_{2}, \cdots, k_{r}\right)$ such that $1 \leqq r \leqq n$, $1 \leqq k_{i} \leqq n$ for all $i \in[1, r]$, and $k_{1}+k_{2}+\cdots+k_{r}=n$. Since $a_{n}$ is one of those summands, $\mathfrak{a}_{n} \subseteq \mathfrak{b}_{n}$. Thus $\mathfrak{b}_{n}$ is an open ideal of $A$. Clearly $\mathfrak{b}_{n} \mathfrak{b}_{m} \subseteq$ $\mathfrak{b}_{n+m}$ for all $n, m \geqq 1$. If $n \geqq m$, then $\mathfrak{b}_{n} \subseteq \mathfrak{b}_{m}$, for if $k_{1}+\cdots+k_{r}=n$, we may find, for some $s \leqq r$, positive integers $j_{1}, \cdots, j_{s}$ such that $j_{i} \leqq k_{i}$ for all $i \in[1, s]$ and $j_{1}+\cdots+j_{s}=m$, whence $\mathfrak{a}_{k_{1}} \cdots \mathfrak{a}_{k_{r}} \subseteq \mathfrak{a}_{j_{1}} \cdots \mathfrak{a}_{j_{s}} \subseteq \mathfrak{b}_{m}$. We shall next show that $\mathfrak{b}_{n^{2}} \subseteq \mathrm{r}^{n}+\mathfrak{a}_{n+1}$. Let $k_{1}+\cdots+k_{r}=n^{2}$. If each $k_{i} \leqq n$, then $n^{2}=k_{1}+\cdots+k_{r} \leqq n r$, so $n \leqq r$, whence $\mathfrak{a}_{k_{1}} \cdots \mathfrak{a}_{k_{r}} \subseteq \mathfrak{r}^{r} \subseteq \mathfrak{r}^{n}$. If $k_{i} \geqq n+1$ for some $i$, then $\mathfrak{a}_{k_{1}} \cdots \mathfrak{a}_{k_{r}} \subseteq \mathfrak{a}_{k_{i}} \subseteq \mathfrak{a}_{n+1}$. Therefore $b_{n^{2}} \subseteq \mathfrak{r}^{n}+\mathfrak{a}_{n+1}$. Given $m$, let $n$ be so large that $n+1 \geqq m$ and $\mathfrak{r}^{n} \subseteq \mathfrak{a}_{m}$; then $\mathfrak{b}_{n^{2}} \subseteq \mathfrak{a}_{m}$. Hence $\left\{\mathfrak{b}_{n}\right\}_{n \geqq 1}$ is a fundamental system of ideal neighborhoods of zero of $A$ satisfying $\mathfrak{b}_{n} \mathfrak{b}_{m} \subseteq \mathfrak{b}_{n+m}$ for all $n, m \geqq 1$. Therefore the function $v$ defined by $v(x)=0$ if $x \notin b_{1}, v(x)=n$ if $x \in b_{n}$ but $x \notin b_{n+1}, v(0)=+\infty$ is a natural pseudovaluation defining the topology of $A$.

$A$ topologically artinian ring is a Hausdorff topological ring $A$ such that the open left ideals of $A$ form a fundamental system of neighborhoods of zero and for every open left ideal $\mathfrak{a}$, the left $A$-module $A / \mathfrak{a}$ is artinian (it is not necessary to assume here that $A$ has an identity element). The complete topologically artinian rings are precisely the strictly linearly compact rings [2, p. 111] (called "im engeren Sinne linear kompakt" rings in [5]).

THEOREM 2. If $A$ is a topologically artinian ring and if every open left ideal contains an open ideal, then the radical $\mathrm{r}$ of $A$ is topologically nilpotent.

Proof. Let $\mathfrak{b}$ be an open ideal of $A$; then $A / \mathfrak{b}$ is an artinian ring and hence has a nilpotent radical. Since $(\mathfrak{r}+\mathfrak{b}) / \mathfrak{b}$ is contained in the radical of $A / \mathfrak{b}$, we conclude that $\mathfrak{r}^{n} \subseteq \mathfrak{b}$ for some $n \geqq 1$. Thus $\mathfrak{r}$ is topologically nilpotent.

A norm is bounded if its values are contained in a bounded interval.

THEOREM 3. Let $A$ be a strictly linearly compact ring, and let $\mathfrak{r}$ be the radical of $A$. The following statements are equivalent:

$1^{\circ}$ The topology of $A$ is defined by a bounded, nonarchimedean norm.

$2^{\circ}$ The topology of $A$ is defined by a norm, and every open left ideal contains an open ideal.

$3^{\circ} A$ is metrizable, $\mathfrak{r}$ is open, and every open left ideal contains an open ideal.

$4^{\circ}$ The topology of $A$ is defined by a natural pseudovaluation.

Proof. For a given norm, let $B_{s}$ denote the closed ball of center 0 and radius $s$. To show that $1^{\circ}$ implies $2^{\circ}$, let \|\| be a nonarchimedean 
norm satisfying $\|x\| \leqq c$ for all $x \in A$, where $c \geqq 1$. Then for any $t>0$, the ideal generated by $B_{t / c^{2}}$ is contained in $B_{t}$, since $\|a x\| \leqq t / c,\|x b\| \leqq t / c$, and $\|a x b\| \leqq t$ for all $x \in B_{t / c^{2}}$ and all $a, b \in A$. Thus $2^{\circ}$ holds.

If $2^{\circ}$ holds, then $B_{1 / 2}$ contains an open ideal $n$; clearly $\lim x^{n}=0$ for all $x \in \mathfrak{n}$, so $\mathrm{n} \subseteq \mathfrak{r}$ by [4, Corollary, Theorem 12] as $A$ is complete, whence $3^{\circ}$ holds. By Theorems 1 and $2,3^{\circ}$ implies $4^{\circ}$, and clearly $4^{\circ}$ implies $1^{\circ}$.

COROLlary 1. Conditions $1^{\circ}, 2^{\circ}$, and $4^{\circ}$ of Theorem 3 are equivalent for a topologically artinian ring.

PROOF. We need only apply Theorem 3 to the completion of a topologically artinian ring, since the completion is clearly strictly linearly compact.

COROLLARY 2 Let $A$ be a totally disconnected compact ring, and let $\mathfrak{r}$ be the radical of $A$. The following statements are equivalent:

$1^{\circ}$ The topology of $A$ is defined by a norm.

$2^{\circ} A$ is metrizable and $\mathfrak{r}$ is open.

$3^{\circ}$ The topology of $A$ is defined by a natural pseudovaluation.

Proof. The open ideals of $A$ form a fundamental system of neighborhoods of zero [4, Lemma 9], so $A$ is strictly linearly compact since $A / \mathfrak{b}$ is a finite ring for any open ideal $\mathrm{b}$.

A compact ring with identity is totally disconnected, [4, Theorem 8] and if it has no divisors of zero, its radical is open [4, Theorem 19]. Hence Corollary 2 generalizes [6, Theorem 7].

Here is an example of a commutative, metrizable, linearly compact ring with open radical whose topology is not given by a norm: Let $A=K[[X, Y]]$, the ring of formal power series in two variables over a field $K$. Since $A$ is a local noetherian domain complete for the topology defined by the powers of its radical $\mathrm{r}=(X)+(Y), A$ is linearly compact for the discrete topology [7, pp. 271-272] and hence for any weaker, Hausdorff linear topology (cf. [2, Exercises 14-20, pp. 108-111], [5]). Moreover, $A$ is a unique factorization domain [7, Theorem 6, p. 148]. Let $\left(p_{n}\right)_{n \geqq 1}$ be a sequence of irreducible elements no two of which are associates (e.g., let $p_{n}=X+Y^{n}$ ), let $a_{n}$ be the principal ideal generated by $p_{1} \cdots p_{n}$, and let $\mathscr{T}$ be the topology on $A$ for which $\left\{a_{n}\right\}_{n \geqq 1}$ is a fundamental system of neighborhoods of zero. As $A$ is a unique factorization domain, $\bigcap_{n \geqq 1} \mathfrak{a}_{n}=(0)$; as $A$ is local, $\mathfrak{a}_{1} \subseteq \mathfrak{x}$. Equipped with topology $\mathscr{T}$, therefore, $A$ is a commutative, linearly compact, metrizable ring with open radical. If $\mathscr{T}$ were defined by a norm, then $\left\{x \in A: \lim x^{n}=0\right\}$ would be a neighborhood of zero and hence would contain $a_{m}$ for some $m$; therefore $\lim _{n}\left(p_{1} \cdots p_{m}\right)^{n}=0$, and in particular, $\left(p_{1} \cdots p_{m}\right)^{n} \in \mathfrak{a}_{m+1}$ for 
some $n$, whence $p_{m+1}$ would divide $p_{1}^{n} \cdots p_{m}^{n}$, a contradiction of the fact that $A$ is a unique factorization domain.

We note finally that P. M. Cohn's criterion for the normability of a topological field may be extended to topological rings. A subset $B$ of a topological ring is bounded if for every neighborhood $V$ of zero there is a neighborhood $U$ of zero such that $U B \subseteq V$ and $B U \subseteq V$. Our proof of the following theorem is a simplification of proofs in [3].

THEOREM 4. Let $A$ be a Hausdorff topological ring whose center contains a cancellable element $a$ such that $x \mapsto a x$ is an open mapping and $\lim a^{n}=0$. If $A$ has a bounded neighborhood of zero [a bounded, open additive subgroup], then the topology of $A$ is defined by a norm [a nonarchimedean norm].

Proof. Let $A_{1}$ be the rings of all the fractions $x / y$, where $x \in A$ and where $y$ is a cancellable element of $A$ belonging to the center of $A$ such that $z \mapsto z y$ is an open mapping, topologized by declaring the neighborhoods of zero in $A$ a fundamental system of neighborhoods of zero in $A_{1}$. Clearly $A_{1}$ is a topological ring containing $A$ as an open subring; consequently, replacing $A$ with $A_{1}$, if necessary, we may assume that $a$ is invertible in $A$.

Let $V$ be a bounded neighborhood of zero. Replacing $V$ by $V \cap(-V)$, if necessary, we may assume that $V$ is symmetric. Let $U=\{x \in A: x V \subseteq V\}$. As $V$ is symmetric and bounded, $U$ is a symmetric neighborhood of zero. To show that $U$ is bounded, let $W$ be a neighborhood of zero. As $V$ is bounded, there exists a neighborhood $T$ of zero such that $T V \subseteq W$, $V T \subseteq W ;$ as $\lim a^{n}=0, a^{k} \in V$ for some $k$; hence $a^{k} T$ is a neighborhood of zero, and $U a^{k} T \subseteq V T \subseteq W$, and similarly $a^{k} T U=T U a^{k} \subseteq W$. Thus $U$ is a bounded symmetric neighborhood of zero that clearly satisfies $U U \subseteq U$. Let $U_{1}$ be a neighborhood of zero such that $U_{1} \subseteq U$ and $U_{1} U+U_{1} U+$ $U_{1} U \subseteq U$; replacing $a$ by a power of $a$, if necessary, we may assume that $a \in U_{1}$; then $a U+a U+a U \subseteq U$. Clearly $\left(a^{k} U\right)_{k \in Z}$ is a decreasing sequence of neighborhoods of zero; it is a fundamental system of neighborhoods of zero, for if $Y$ is a neighborhood of zero, there exists a neighborhood $Z$ of zero such that $Z U \subseteq Y$ as $U$ is bounded, and there exists $t \geqq 0$ such that $a^{t} \in Z$ since $\lim a^{n}=0$, whence $a^{t} U \subseteq Z U \subseteq Y$. In particular, $\bigcap_{k \geqq 0} a^{k} U=(0)$.

Let $g(0)=0$, and for each nonzero $x \in A$ let $g(x)=2^{-k}$, where $k$ is the largest of the integers $j$ such that $x \in a^{j} U$ (such an integer exists, for as $\lim a^{n} x=0, x \in a^{-n} U$ for some $n \geqq 0$ ). If $V$ is an additive subgroup, then $U$ is a subring, and $g$ is clearly a nonarchimedean norm defining the topology of $A$.

In general, let $f(x)=\inf \left\{\sum_{i=1}^{p} g\left(z_{i}\right): z_{1}+\cdots+z_{p}=x\right\}$. Since $a U+$ $a U+a U \subseteq U, a^{n+1} U+a^{n+1} U+a^{n+1} U \subseteq a^{n} U$ for all $n \in \mathrm{Z}$; by the argument 
of [1, Proposition 2, p. 15], $f$ is a norm defining the topology of the additive group $A$ (by induction on $p$, one shows that $\frac{1}{2} g\left(\sum_{i=1}^{p} z_{i}\right) \leqq$ $\left.\sum_{i=1}^{p} g\left(z_{i}\right)\right)$. Since $a \in U$ and $U U \leqq U, g(x y) \leqq g(x) g(y)$ for all $x, y \in A$, whence $f(x y) \leqq f(x) f(y)$; thus $f$ is a norm defining the topology of the ring $A$.

COROllary [3, TheOREMS 6.1 AND 7.1]. The topology of an indiscrete, Hausdorff topological field $K$ is defined by a norm [a nonarchimedean norm] if and only if there is a nonzero element $a$ of $K$ such that $\lim a^{n}=0$ and $K$ contains $a$ bounded neighborhood of zero [a bounded, open additive subgroup].

\section{REFERENCES}

1. N. Bourbaki, Eléments de mathématique. I: Les structures fondamentales de l'analyse. Fasc. VII, Livre III: Utilisation des nombres réels en topologie générale, 2ième éd., Actualités Sci. Indust., no. 1045, Hermann, Paris, 1958. MR 30 \#3439.

2. —_, Eléments de mathénaatique. Fasc. XXVIII. Algèbre commutative. Chaps. 3, 4, Actualités Sci. Indust., no. 1293, Hermann, Paris, 1961. MR 30 \#2027.

3. P. M. Cohn, An invariant characterization of pseudo-valuations on a field, Proc. Cambridge Philos. Soc. 50 (1954), 159-177. MR 16, 214.

4. Irving Kaplansky, Topological rings, Amer. J. Math. 69 (1947), 153-183. MR 8, 434.

5. Horst Leptin, Linear kompakte Moduln und Ringe, Math. Z. 62 (1955), 241-267. MR 16, 1085.

6. Z. S. Lipkina, Locally bicompact rings without zero divisors, Izv. Akad. Nauk SSSR Ser. Mat. 31 (1967), 1239-1262=Math. USSR Izv. 1 (1967), 1187-1208. MR 36 \#2657.

7. Oscar Zariski and Pierre Samuel, Commutative algebra. Vol. II, The University Series in Higher Math., Van Nostrand, Princeton, N.J., 1960. MR 22 \#11006.

Department of Mathematics, Duke University, Durham, North Carolina 27706 